ANNALS OF “DUNAREA DE JOS” UNIVERSITY OF GALATI
MATHEMATICS, PHYSICS, THEORETICAL MECHANICS
FASCICLE II, YEAR XIII (XLIV) 2021, No. 1
DOI: https://doi.org/10.35219/ann-ugal-math-phys-mec.2021.1.09

\title{
Study on the chemical potential of apigenin, luteolin, quercetin, and myricetin using the molecular modeling
}

\author{
Steluța Gosav ${ }^{1, *}$, Adriana Hodorogea ${ }^{2}$, Dan Maftei ${ }^{3}$ \\ 1 "Dunarea de Jos" University of Galati, Faculty of Sciences and Environment, INPOLDE research center, Re- \\ FORM-UDJG Platform, 800008 Galati, Romania \\ 2"Dunărea de Jos" University of Galati, Faculty of Medicine and Pharmacy, 35 Al. I. Cuza Street, Galati, \\ Romania \\ 3“Al.I. Cuza” University, Chemistry Department, 11 Carol I Bldv. RO-700506, Iasi, Romania \\ *Corresponding author: sgosav@ugal.ro
}

\begin{abstract}
In the present paper, the chemical potential of four flavonoids i.e. apigenin, luteolin, quercetin, and myricetin, of interest in the pharmaceutical industry was investigated using molecular modelling. The equilibrium geometry of molecular structures was calculated in the gas phase and ground state by using B3LYP hybrid functional in conjunction with a 6-311G(d,p) basis set. In order to assess the chemical potential of investigated flavonoids, the main quantum molecular descriptors, such as the dipole moment, the energy of the highest/lowest occupied/unoccupied molecular orbital, the gap energy, the electronegativity, the chemical hardness/softness, and the electrophilicity index have been computed. Also, the influence of the hydroxylation degree of chemical compounds on the chemical potential is discussed.
\end{abstract}

Keywords: flavonoids, DFT, chemical potential, molecular modeling.

\section{INTRODUCTION}

The flavonoids are an important chemical class of bioactive products, found in several fruits, vegetables, herbs, and spices which possess a wide range of pharmacological properties, such as antioxidant, anti-inflammatory, anticancer, neuroprotective, cardioprotective, immunomodulatory properties, etc. [1,2]. It is known that the oxidant compounds generate many important diseases in the human body by the fact that they participate in the formation of many cell toxins. For this reason, antioxidant compounds and functional foods that possess antioxidant properties have gained great usage in the prevention of oxidation-associated diseases such as cardiovascular diseases, diabetes, and cancer. The flavonoids are antioxidant compounds with a potent scavenger of reactive oxygen/nitrogen species due to their antioxidant pharmacophores for free-radical scavenging [3].

In the last years, among the flavonoids that were mostly investigated with important antioxidant properties are the apigenin (4',5,7-trihydroxyflavone), luteolin $\left(3^{\prime}, 4^{\prime}, 5,7-\right.$ tetrahydroxyflavone), quercetin $\left(3,3^{\prime}, 4^{\prime}, 5,7\right.$-pentahydroxyflavone) and myricetin $\left(3,3^{\prime}, 4^{\prime}, 5,5^{\prime}, 7-\right.$ hexahydroxyflavone) [1-7]. Chemically, these compounds have a C6-C3-C6 structure that contains two benzene rings and one oxygen containing ring with a $\mathrm{C} 2-\mathrm{C} 3$ carbon double bond (see Fig. 1). The apigenin, luteolin, quercetin, and myricetin are mainly present in the form of sugar conjugated derivative in different vegetables, fruits, and medicinal plants $[3,7]$. 
<smiles></smiles>

Fig. 1. Chemical structure of investigated flavonoids

In this paper, four flavonoids (apigenin, luteolin, quercetin, and myricetin) have been investigated from a chemical reactivity point of view using density functional theory (DFT). For this purpose, we have evaluated some electronic parameters computed at B3LYP/6-311G(d,p) level.

\section{COMPUTATIONAL DETAILS}

The geometrical parameters (bond lengths and angles) and IR frequencies of studied flavonoids have been computed by using B3LYP hybrid functional in correlation with $6-311 \mathrm{G}(\mathrm{d}, \mathrm{p})$ basis set with Gaussian 09 program [8]. The computations were carried out in the ground state and gas phase for all compounds. As there are no imaginary IR frequencies it means that the optimized geometry is located at the minimum on the potential energy surface.

The steps followed in the computational procedure are:

- $\quad$ for each compound, we have created a .com file using GaussView 5.09. software [9];

- the .com file has been used as input for the Gaussian 09 software, which performs the optimization of the molecular structures and computes the IR frequencies;

- the output files of the Gaussian 09 program are the .chk file and the $\log$ file. The. $\log$ file has been used by the GaussView 5.09. program in order to visualize the optimized molecular structures. Also, from the $\log$ file, it was extracted some parameters namely, the dipole moment (DM), the highest occupied molecular orbital (HOMO), and the lowest unoccupied molecular orbital (LUMO).

- the electronegativity $(\chi)$, the chemical hardness $(\eta)$ /softness $(\sigma)$, the electrophilicity index $(\omega)$ and gap energy $\left(E_{g a p}\right)$ were calculated using the formulas shown in a previous paper [10].

\section{RESULTS AND DISCUSSION}

The optimized molecular structures and atom numbering of the investigated flavonoids are shown in Fig. 2. Regarding the spatial orientation of the optimized molecular structures of apigenin and luteolin we can mention the following: the dihedral angle (C8-C9-C11-C12) between the ring B and ring $\mathrm{A}$ is small, its value being $16.55^{\circ}$ in the case of apigenin and $18.2^{\circ}$ in the case of luteolin, between the hydrogen atom, $\mathrm{H} 26$, of the hydroxyl group $(\mathrm{O} 20-\mathrm{H} 26)$ at the $\mathrm{C} 5$ position of the benzopyran structure and the oxygen atom, $\mathrm{O} 18$, of the $\mathrm{C}=\mathrm{O}$ carbonyl group $(\mathrm{C} 7=\mathrm{O} 18)$ of the same structure appeared an intramolecular hydrogen bond (IHB) with the length of $1,7 \AA$ in the case of apigenin and 1,69 $\AA$ in the case of luteolin (see Fig.1 a) and b)).

The molecular structures of quercetin and myricetin are almost planar, the dihedral angle between the ring B and ring A being extremely small (C8-C9-C11-C12) namely $0.07^{\circ}$ in the case of quercetin and $0.06^{\circ}$ in the case of myricetin (see Fig.1 c) and d)). Also, these molecular structures present two IHBs between $\mathrm{H} 29$ hydrogen atom respectively $\mathrm{H} 31$, of the hydroxyl group from $\mathrm{C} 3$ respectively $\mathrm{C} 5$ position of the benzopyran structure and $\mathrm{O} 18$ oxygen atom of the carbonyl group (C7 $=\mathrm{O} 18$ ) of the same structure. Analyzing the 3D molecular structure of studied flavonoids we mention that the quercetin and myricetin have planar molecular structures due to the presence of hydroxyl chemical group at the $\mathrm{C} 3$ position of the benzopyran structure.

The theoretical electronic parameters shown in Table 1 provide information about the distribution of electrons in the molecule and can be used to estimate chemical reactivity. 


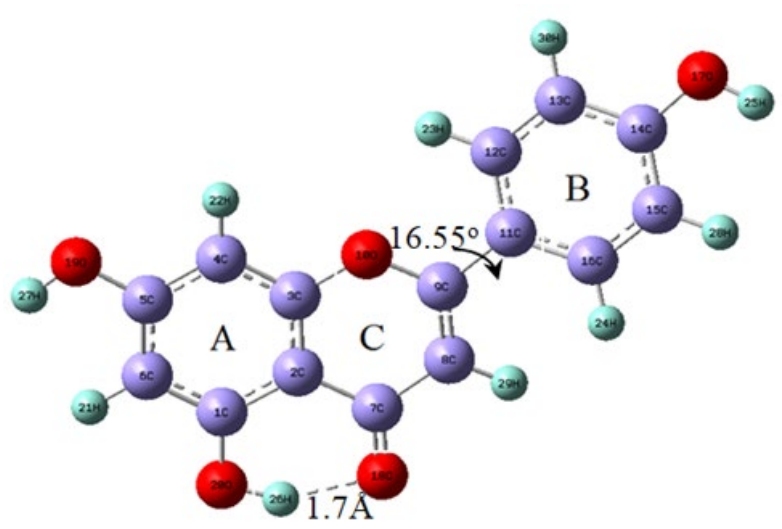

a)

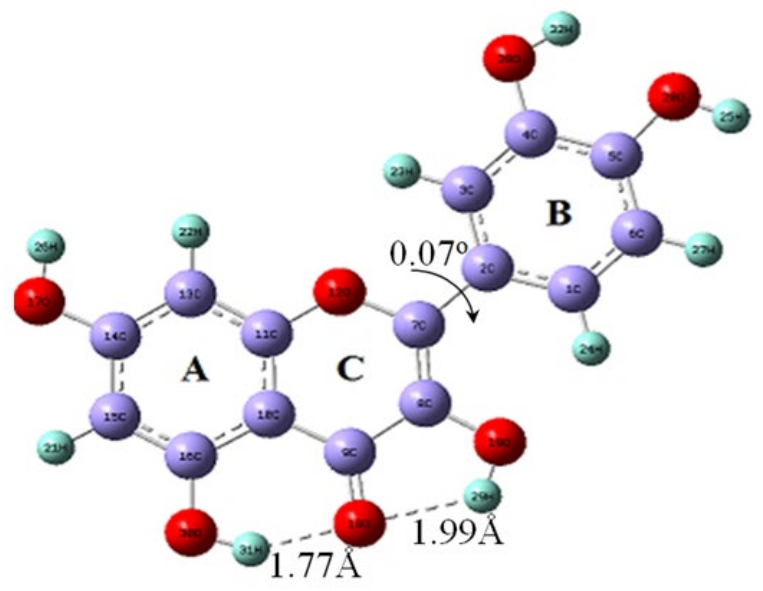

c)

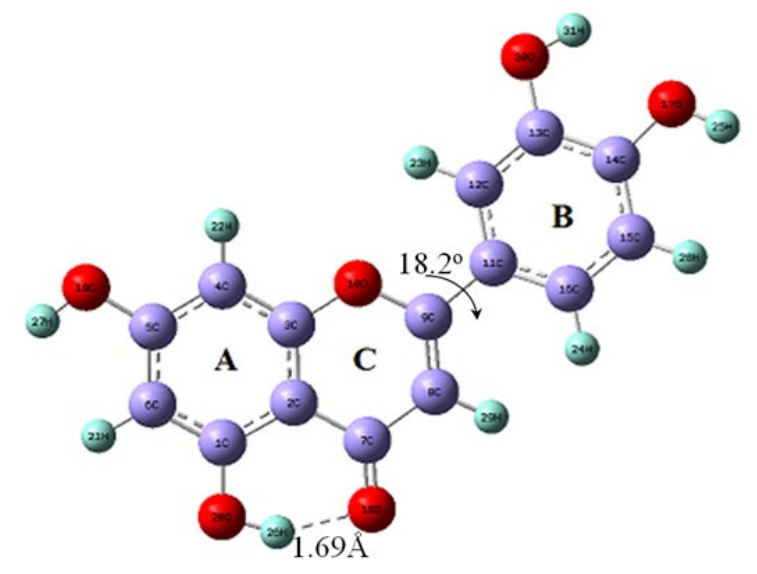

b)

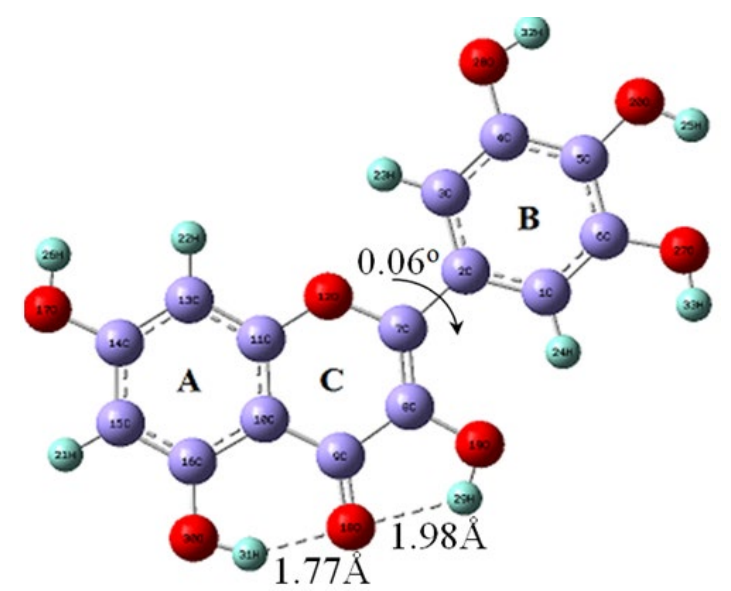

d)

Fig. 2. Optimized molecular structure of apigenin (a), luteolin (b), quercetin (c,) and myricetin (d)

A very important parameter that is responsible for kinetic stability and chemical reactivity is the gap energy. Analyzing Table 1, we observed that the $E_{\text {gap }}$ energy decreases with the increase of the hydroxylation degree of the investigated flavonoids. Thus, the apigenin, which has three hydroxyl groups, has the highest value of Egap i.e. $4.20 \mathrm{eV}$, and the myricetin with six hydroxyl groups, has the smallest value of Egap, i.e. $3.71 \mathrm{eV}$. Also, we have remarked that the $\mathrm{E}_{\text {gap }}$ energy decreases more when it passes from flavones (apigenin - $4.20 \mathrm{eV}$ and luteolin $-4.13 \mathrm{eV}$ ) to flavonols (quercetin - $3.74 \mathrm{eV}$ and myricetin $-3.71 \mathrm{eV}$ ). An explanation of this fact may be the presence of the $-\mathrm{OH}$ chemical group at position 3 of the benzopyran structure for flavonols.

It is known that a small (high) energy gap is responsible for low (high) kinetic stability and high (low) chemical reactivity [10]. In our case, it is worth emphasizing the fact that the flavonols have lower kinetic stability and higher chemical reactivity than flavones.

Table 1. Gibbs free energy and some electronic parameters of investigated compounds

\begin{tabular}{|c|c|c|c|c|c|c|c|c|c|}
\hline $\begin{array}{c}\text { Compound } \\
\text { name }\end{array}$ & $\begin{array}{c}\mathrm{G}^{\mathrm{o}} \\
(\text { Hartree })\end{array}$ & $\begin{array}{c}\mathrm{E}_{\text {LUMO }} \\
(\mathrm{eV})\end{array}$ & $\begin{array}{c}\mathrm{E}_{\text {HOMO }} \\
(\mathrm{eV})\end{array}$ & $\begin{array}{c}\mathrm{E}_{\text {gap }} \\
(\mathrm{eV})\end{array}$ & $\begin{array}{c}\eta \\
(\mathrm{eV})\end{array}$ & $\begin{array}{c}\sigma \\
(\mathrm{eV})^{-1}\end{array}$ & $\begin{array}{c}\chi \\
(\mathrm{eV})\end{array}$ & $\begin{array}{c}\omega \\
(\mathrm{eV})\end{array}$ & $\begin{array}{c}\mathrm{DM} \\
(\text { debye })\end{array}$ \\
\hline apigenin & -953.82 & -1.97 & -6.17 & 4.20 & 2.1 & 0.24 & 4.07 & 3.95 & 3.83 \\
\hline luteolin & -1029.06 & -1.99 & -6.12 & 4.13 & 2.06 & 0.24 & 4.05 & 3.98 & 4.85 \\
\hline quercetina & -1104.30 & -2.05 & -5.79 & $\mathbf{3 . 7 4}$ & $\mathbf{1 . 8 7}$ & $\mathbf{0 . 2 7}$ & 3.93 & $\mathbf{4 . 1 2}$ & 5.06 \\
\hline myricetin & -1179.54 & -2.10 & -5.81 & $\mathbf{3 . 7 1}$ & $\mathbf{1 . 8 5}$ & $\mathbf{0 . 2 7}$ & 3.95 & $\mathbf{4 . 2 1}$ & 3.77 \\
\hline
\end{tabular}


The values of the chemical hardness and chemical softness supply us with the same information regarding the stability/reactivity of compounds. Thus, the flavonols, which have a lower chemical hardness and higher chemical softness, present lower kinetic stability and higher chemical reactivity than flavones. Regarding the electrophilicity index (see Table 1), we remarked that all compounds have a strong electrophilic character, the flavonols being slightly more electrophiles than flavones.

In drug design, an improved dipole moment has an important role to increase the binding affinity between the ligand and its receptor by the enhancement of hydrogen bond and non-bonded interactions [11]. In our case, of all studied compounds, quercetin has the highest dipole moment with 5.064 debye.

\section{CONCLUSIONS}

The chemical potential of investigated flavonoids is very influenced by the hydroxylation degree of molecular structures and by the $-\mathrm{OH}$ group at position 3 of the benzopyran structure. Thus, the flavonols, which are present at position 3 of the benzopyran structure a hydroxyl chemical group, present lower kinetic stability/higher chemical reactivity than flavones which do not have the $-\mathrm{OH}$ group at position 3 of the benzopyran structure. The most hydroxylated compound namely, myricetin, is the flavonoid with the lowest kinetic stability and highest chemical reactivity, respectively.

\section{References}

1. Gosav S., Birsa M.L., Multivariate study of flavonoids active against Caco-2 colon carcinoma, Romanian Reports in Physics 66(2) (2014) 411-426.

2. Paduraru N., Gosav S., Praisler M., Pharmacological Action of Flavonoids - An Overview, Annals of "Dunarea de Jos" University of Galati, Mathematics, physics, theoretical mechanics, fascicle II, year VI (XXXVII) 1 (2014) 63-73.

3. Salehi B., Machin L., Monzote L., Sharifi-Rad J., Ezzat S.M., Salem M.A., Merghany R.M., El Mahdy N.M., Kılı̨̧ C.S., Sytar O., Sharifi-Rad M., Sharopov F., Martins N., Martorell M., Cho W.C., Therapeutic Potential of Quercetin: New Insights and Perspectives for Human Health, ACS Omega 5(20) (2020) 11849-11872.

4. Lefort EC, Blay J. Apigenin and its impact on gastrointestinal cancers. Mol Nutr Food Res 57 (2013) 126-144.

5. Ginwala R., McTish E., Raman C., Singh N., Nagarkatti M., Nagarkatti P., Sagar D., Jain P., Khan Z.K., Apigenin, a Natural Flavonoid, attenuates EAE severity through the modulation of dendritic cell and other immune cell functions, J Neuroimmune Pharmacol 11 (2016) 36-47.

6. Nabavi S.F., Braidy N., Gortzi O., Sobarzo-Sanchez E., Daglia M., Skalicka-Wozniak K., Nabavi S.M., Luteolin as an anti-inflammatory and neuroprotective agent: a brief review, Brain Res Bull 119 (2015) 1-11.

7. Taheri Y., Suleria H.A.R., Martins N. Sytar O., Beyatli A., Yeskaliyeva B., Seitimova G., Salehim B., Semwal P., Painuli S., Kumar A., Azzini E., Martorell M., Setzer W.N., Maroyi A., Sharifi-Rad J., Myricetin bioactive effects: moving from preclinical evidence to potential clinical applications, BMC Complement Med Ther 20 (2020) 1-14.

8. Gaussian 09 software package, Revision D.01, Frisch M.J., Trucks G.W. et al., Gaussian, Inc., Wallingford CT, 2009.

9. GaussView, version 5.09, R. Dennington T., Keith J., Millam, Semichem Inc., Shawnee Mission, KS, 2009.

10. Gosav S, Paduraru N, Maftei D, Birsa ML, Praisler M., Quantum chemical study of a derivative of 3-substituted dithiocarbamic flavanone, Spectrochimica Acta Part A: Molecular and Biomolecular Spectroscopy 172 (2017) 115-125.

11. Moniruzzaman, Hoque M.J., Physiochemical, molecular docking, and pharmacokinetic studies of Naproxen and its modified derivatives based on DFT, International Journal of Scientific Research and Management 6(9) (2018) 12-19. 